\title{
9. Tratamiento farmacológico en la fibrosis pulmonar idiopática. Drogas modificadoras de enfermedad
}

\author{
MATÍAS FLORENZANO V.* y FELIPE REYES C.**
}

\section{Pharmacological treatment in the FPI. Disease modifying drugs}

The natural history of idiopathic pulmonary fibrosis (IPF) is heterogeneous and unpredictable. The course of the disease without treatment, is inevitably progressive, with a poor prognosis. Historical treatments have varied from corticosteroids and immunosuppressants (azathioprine, cyclophosphamide), to colchicine and $\mathrm{N}$-acetylcysteine. In the last decades, multiple failed therapeutic trials have been carried out. However, since 2014 in the United States, Europe and other countries, two drugs, called antifibrotic therapy or disease modifiers, are approved for the treatment of IPF: nintedanib and pirfenidone. The purpose of antifibrotic therapy is to slow down the decline in lung function in patients with IPF up to $50 \%$.

Key words: Idiopathic Pulmonary Fibrosis; pirfenidone; nintedanib; Immunosuppressive agents.

\section{Resumen}

La historia natural de la fibrosis pulmonar idiopática (FPI) es heterogénea e impredecible. Aunque el curso de la enfermedad, sin tratamiento, es inevitablemente progresiva y de mal pronóstico. Los tratamientos históricos han variado desde corticosteroides e inmunosupresores (azatioprina, ciclofosfamida), hasta colchicina y $N$-acetilcisteína. En las últimas décadas se han realizado múltiples ensayos terapéuticos fallidos. Sin embargo, desde el año 2014 en los Estados Unidos, Europa y otros países, dos drogas, denominadas terapia antifibrótica o modificadoras de la enfermedad, están aprobadas para el tratamiento de la FPI: nintedanib y pirfenidona. La terapia antifibrótica, tiene como objetivo enlentecer en hasta $50 \%$ la declinación de la función pulmonar en pacientes con FPI.

Palabras clave: Fibrosis pulmonar idiopática; pirfenidona; nintedanib; Agentes Inmunosupresores.

La historia natural de la fibrosis pulmonar idiopatica (FPI) es heterogénea e impredecible ${ }^{1}$. Aunque el curso de la enfermedad, sin tratamiento, es inevitablemente progresiva, con mal pronóstico.

Una vez hecho el diagnóstico, el tratamiento debe ser iniciado de forma oportuna, siendo fundamental preservar la función pulmonar y reducir los riesgos de exacerbaciones agudas ${ }^{2}$.

Los tratamientos históricos han variado desde corticosteroides e inmunosupresores (azatioprina, ciclofosfamida) hasta colchicina y $\mathrm{N}$-acetilcisteína.

En las últimas décadas se han realizado múltiples ensayos terapéuticos fallidos. El estudio IFIGENIA evaluó la eficacia de $\mathrm{N}$-acetilcisteína
(NAC), una sustancia precursora del antioxidante glutatión, en combinación con prednisona y azatioprina. Esta "triple terapia" redujo la disminución de función pulmonar al año en comparación con prednisona y azatioprina sola ${ }^{3}$. Sin embargo, la falta de una rama placebo, condujo a una evaluación posterior que fue el estudio PANTHER. Este fue un estudio aleatorizado, controlado con placebo, de tres ramas ${ }^{4}$, que comparó la terapia combinada con prednisona, azatioprina y NAC, con NAC sola y placebo. La "triple terapia" se asoció con un aumento significativo en la mortalidad por todas las causas, hospitalizaciones y eventos adversos graves. Hoy en día se considera un tratamiento perjudicial para los pacientes con

\footnotetext{
* Instituto Nacional del Tórax y Clínica Las Condes. Santiago,Chile.

** Coordinador Comisión de Enfermedades pulmonares intersticiales difusas, SER Chile. Hospital Clínico Universidad de Chile, Instructor Universidad de Chile. Santiago, Chile.
} 
FPI. La rama NAC contra placebo se publicó posteriormente y no mostró efectos adversos graves, pero tampoco beneficios significativos en la reducción la función pulmonar ${ }^{5}$.

El sildenafil se ha estudiado en dos ensayos controlados aleatorizados en pacientes con $\mathrm{FPI}^{6,7}$. En el estudio STEP-IPF, no se observaron beneficios para el desenlace primario: cambios en los metros recorridos en el test de caminata en $6 \mathrm{~min}$, pero sí beneficios en algunos desenlaces secundarios en un subgrupo de pacientes, con disfunción de cavidades cardíacas derechas (DCD). En el segundo estudio, no hubo beneficio alguno. Recientemente se ha evaluado la eficacia de sildenafil en combinación con nintedanib en el estudio INSTAGE ${ }^{8}$ en pacientes con FPI avanzada (DLCO $<35 \%$ ), cuyos resultados fueron negativos.

Desde el año 2014 en los Estados Unidos, Europa y otros países, dos drogas, denominadas terapia antifibrótica o modificadoras de la enfermedad, están aprobadas para el tratamiento de la FPI: nintedanib y pirfenidona.

La pirfenidona fue el primer fármaco aprobado para el tratamiento de la FPI. Es una molécula pleiotrópica con propiedades antifibróticas, antiinflamatorias y antioxidantes. Se ha demostrado que bloquea las principales vías de transducción de señales relevantes para la fibrosis, incluidas las del factor de crecimiento transformante beta (TGF- $\beta$ ), el factor de crecimiento de fibroblastos y la interleuquina uno beta (IL-1 $\beta)^{9}$.

Nintedanib fue aprobado el 2014 para el tratamiento de la FPI. Es un inhibidor intracelular de las tirosina quinasas, incluido el factor de crecimiento endotelial vascular, el factor de crecimiento de fibroblastos y los receptores del factor de crecimiento derivado de plaquetas ${ }^{10}$. Nintedanib inhibe la transformación de fibroblastos inducidos por TGF- $\beta$ a miofibroblastos y la liberación de colágeno a partir de fibroblastos pulmonares estimulados por TGF- $\beta$.

La terapia antifibrótica, tiene como objetivo enlentecer la declinación de la función pulmonar, que se estima puede ocurrir en sobre el $90 \%$ de los pacientes en el transcurso de un año post diagnóstico ${ }^{11}$. La capacidad vital forzada (CVF) cae en promedio entre $150-200 \mathrm{ml} /$ año en pacientes con FPI ${ }^{12}$, premisa válida también para pacientes que presentan función pulmonar conservada en el momento del diagnóstico.

Los estudios INPULSIS ${ }^{13}$ y ASCEND $^{14}$ demostraron que, en sujetos con deterioro leve o moderado de la CVF, nintedanib y pirfenidona lograron enlentecer la caída de la CVF en hasta un $50 \%$ durante el primer año de tratamiento.
Los efectos demostrados de ambas drogas han sido consistentes a través de todos los subgrupos estudiados (edad, raza, género, uso concomitante de drogas y comorbilidades) ${ }^{15,16}$, incluyendo pacientes con función pulmonar preservada ${ }^{17,18} \mathrm{O}$ muy deteriorada ${ }^{19,20}$.

En cuanto a los síntomas, la terapia antifibrótica no ha demostrado tener impacto en la disnea, tos, ni en la calidad de vida, en los estudios clínicos a 1 año, sin embargo, en registros de la vida real de más largo plazo sugieren cambios positivos en estos aspectos ${ }^{21}$.

En la actualidad el los EEUU, Europa y Latinoamérica alrededor de los $60 \%$ de los pacientes con FPI están recibiendo terapia antifibrótica. Las principales razones para no tratar a los pacientes son la falta de cobertura por seguros de salud de estas costosas drogas y la percepción de una parte de la comunidad médica de que los efectos adversos superan los beneficios clínicos ${ }^{22,23}$.

La mayoría de los efectos adversos de las drogas modificadoras de enfermedad de FPI son de la esfera gastrointestinal. Para nintedanib es diarrea en un alrededor de $60 \%$ de los casos, pero que lleva a descontinuación de la droga en menos de $5 \%{ }^{24}$. Se recomienda hidratación, antidiarreicos como la loperamida y en algunos casos reducción de dosis, en la practica clínica gran parte de los pacientes presenta un cuadro autolimitado.

La pirfenidona se asocia a náuseas, fatiga y fotosensibilidad $^{25}$. La tasa de suspensión definitiva también es baja con su uso después de las comidas, disminución transitoria de dosis y uso de bloqueador solar.

Ambas drogas pueden producir aumento de las enzimas hepáticas, es importante la vigilancia estricta de estas, de forma mensual al menos los primeros tres meses.

El uso combinado de ambas drogas podría ejercer un efecto sinérgico y su perfil de tolerancia parece ser aceptable ${ }^{26}$.

\section{Recomendaciones en base a la evidencia disponible según metodología GRADE}

1. En pacientes con fibrosis pulmonar idiopática, ¿Se debería utilizar pirfenidona en lugar de tratamiento sintomático?

Recomendación 1: La comisión de enfermedades
pulmonares intersticiales difusas de la Sociedad
Chilena de Enfermedades Respiratorias sugiere
utilizar pirfenidona en lugar de tratamiento
sintomático en pacientes con fibrosis pulmonar
idiopática (recomendación condicional, certeza
de la evidencia baja) 


\section{Resumen de la evidencia}

Identificamos 15 revisiones sistemáticas $(\mathrm{RS})^{27-40}$ que contenían 5 estudios controlados aleatorizados (ECA) publicados en 4 artícu$\operatorname{los}^{14,41-43}$ y ningún ECA adicional (Anexo 1, Apéndice 2).

\section{Beneficios}

El uso de pirfenidona en pacientes con fibrosis pulmonar idiopática reduce en $50 \%$ el deterioro de la capacidad vital forzada, comparado con pacientes semejantes que recibieron tratamiento sintomático. Además, aumenta la distancia que los pacientes pueden caminar en el test de caminata en 6 min, podría minimizar las exacerbaciones agudas y disminuir la cantidad de muertos en relación con el grupo con tratamiento sintomático en protocolos bien realizados con gran cantidad de pacientes. De hecho, al comparar el uso de pirfenidona con tratamiento sintomático, por cada 1.000 pacientes, mueren 24 menos en el grupo que recibió pirfenidona (RR 0,53, IC 95\% 0,32 a 0,88), 109 menos tienen menor reducción de los metros caminados en el test de 6 min (RR 0,74 IC $95 \% 0,64$ a 0,86), 27 personas menos no tendrán una exacerbación aguda (RR 0,59 IC95\% 0,19 a 1,84), 115 personas menos no tendrán una disminución $>10 \%$ en su CVF ( RR 0,63 IC95\% 0,47 a 0,85$)$ y 61 personas menos no verán empeorada su fibrosis (RR 0,64 IC95\% 0,5 a 0,83).

\section{Riesgos}

Al utilizar pirfenidona en pacientes con fibrosis pulmonar idiopática, posiblemente aumentarán la cantidad de efectos adversos. Por cada 1.000 pacientes tratados con pirfenidona 8 más posiblemente tendrán eventos adversos serios (RR 1,02 , IC95\% 0,93 a 1,11) y 159 más posiblemente tendrán eventos adversos relacionados a la piel (RR 4,92; IC95\% 2,1 a 11,53).

\section{Justificación de la recomendación}

El panel consideró que los posibles beneficios de la pirfenidona podrían sobrepasar los riesgos, por lo que hizo una recomendación condicional a favor de su uso.

\section{Consideraciones de implementación}

Pirfenidona, se encuentra disponible en Chile en posología de $267 \mathrm{mg}$ y $200 \mathrm{mg}$. La dosis recomendada según los estudios clínicos es de 2.403 $\mathrm{mg}$ al día, por lo que es necesario el uso de 9 a 12 comprimidos por día según posología empleada. Es recomendable aumentar la dosis de forma progresiva para minimizar los efectos adversos, principalmente gastrointestinales. Recomenda- mos un esquema progresivo por semana, por ejemplo: 1 comprimidos $\mathrm{c} / 8 \mathrm{~h}$ la primera semana, 2 comprimidos $\mathrm{c} / 8 \mathrm{~h}$ las segunda semana y 3 comprimidos $\mathrm{c} / 8 \mathrm{~h}$ la tercera semana, en caso de utilizar la posología de $267 \mathrm{mg}$, si se utiliza la posología de $200 \mathrm{mg}$ debe llegar a la cuarta semana a 4 comprimidos $\mathrm{c} / 8 \mathrm{~h}$. Siempre con las comidas.

Luego de haber alcanzado la dosis máxima, se recomienda control de pruebas hepáticas por riesgo a elevación de las enzimas hepáticas y repetir controles mensuales al menos los primeros tres meses del inicio de la terapia y luego cada 3-6 meses.

En general el perfil de seguridad de la droga es aceptable, son los efectos adversos gastrointestinales los mas frecuentes, que pueden disminuirse con un esquema progresivo. Es importante recordar la posibilidad de lesiones de piel por fotosensibilidad, por lo que se debe recomendar a todos los pacientes el uso de protector solar y cobertura de zonas expuestas.

\section{En pacientes con fibrosis pulmonar idiopáti- ca, ¿se debería utilizar Nintedanib en lugar de tratamiento sintomático?}

Recomendación 2: La comisión de enfermedades pulmonares difusas de la Sociedad Chilena de Enfermedades Respiratorias sugiere utilizar Nintedanib en lugar de tratamiento sintomático en pacientes con fibrosis pulmonar idiopática. (recomendación condicional, certeza de la evidencia moderada)

\section{Resumen de la evidencia}

Identificamos ocho $\mathrm{RS}^{27-29,33,35,36,38,40}$ (4, 5 que contenían tres ECA en dos publicaciones ${ }^{13,44} \mathrm{y}$ ningún ECA adicional (ANEXO 1, Apéndice 3).

\section{Beneficios}

El uso de nintedanib en pacientes con fibrosis pulmonar idiopática posiblemente disminuirá la cantidad de muertes y posiblemente aumentará la distancia recorrida en el test de caminata en 6 min.

Por cada 1.000 pacientes que utilizan nintedanib, posiblemente, morirán 23 menos (RR 0,72 IC95\% 0,47 a 1,08) y la distancia recorrida para el test de caminata $6 \mathrm{~min}$, posiblemente, aumentará en $6,32 \mathrm{~m}$ (IC 95\% 27,08 menos a 39,72 más).

\section{Riesgos}

El uso de Nintedanib en pacientes con fibrosis pulmonar idiopática posiblemente disminuirá la cantidad de eventos adversos serios. Por cada 1.000 pacientes en que se utiliza nintedanib, 
posiblemente 3 menos tendrán eventos adversos serios (RR 0,99 IC95\% 0,83 a 1,18).

\section{Justificación de la recomendación}

El panel consideró que los posibles beneficios del nintedanib podrían sobrepasar los riesgos. Sin embargo, el costo de éste es elevado, por lo que se hizo una recomendación condicional a favor del uso de nintedanib.

\section{Consideraciones de implementación}

Nintedanib se encuentra disponible en Chile, con dos posologías, $150 \mathrm{mg}$ y $100 \mathrm{mg}$.

La dosis habitual es un comprimido de 150 mg 2 veces al día, administrado con $12 \mathrm{~h}$ de diferencia aproximadamente. En algunos casos según la tolerancia puede emplearse $100 \mathrm{mg}$ dos veces por día.

Es recomendable tomarlo siempre con alimentos y a la misma hora.

Los efectos secundarios más frecuentes son gastrointestinales: náuseas, vómitos, dolor abdominal, disminución del apetito, pérdida de peso, aumento de las enzimas hepáticas y diarrea, esta última habitualmente auto limitada los primeros tres meses de tratamiento, este efecto puede disminuirse utilizando concomitantemente antidiarreicos.

Al igual que con pirfenidona, se recomienda control de pruebas hepáticas mensualmente los primeros tres meses.

\section{Bibliografía}

1.- LEY B, COLLARD HR, KING TE Jr. Clinical course and prediction of survival in idiopathic pulmonary fibrosis. Am J Respir Crit Care Med 2011; 183: 431-40.

2.- MAHER TM, STREK ME. Antifibrotic therapy for idiopathic pulmonary fibrosis: time to treat. Respir Res 2019; 20: 205.

3.- DEMEDTS M, BEHR J, BUHL R, COSTABEL U, DEKHUIJZEN R, JANSEN HM, et al. High-dose acetylcysteine in idiopathic pulmonary fibrosis. N Engl J Med 2005; 353: 2292-42.

4.- RAGHU G, ANSTROM KJ, KING TE JR, LASKY JA, MARTÍNEZ FJ, et al; Idiopathic Pulmonary Fibrosis Clinical Research Network, Prednisone, azathioprine, and $\mathrm{N}$-acetylcysteine for pulmonary fibrosis. N Engl J Med 2012; 366: 1968-977.

5.- MARTÍNEZ FJ, DE ANDRADE JA, ANSTROM KJ, KING TE JR, RAGHU G; Idiopathic Pulmonary Fibrosis Clinical Research Network. Randomized trial of acetylcysteine in idiopathic pulmonary fibrosis. N Engl J Med 2014; 370: 2093-101.

6.- JACKSON RM, GLASSBERG MK, RAMOS CF,
BEJARANO PA, BUTROUS G, GÓMEZ-MARÍN $\mathrm{O}$, et al. Sildenafil therapy and exercise tolerance in idiopathic pulmonary fibrosis. Lung 2010; 188: 115-23.

7.- ZISMAN DA, SCHWARZ M, ANSTROM KJ, COLLARD HR, FLAHERY HR, et al; Idiopathic Pulmonary Fibrosis Clinical Research Network. A controlled trial of sildenafil in advanced idiopathic pulmonary fibrosis. N Engl J Med 2010; 363: 620-8.

8.- KOLB M, RAGHU G, WELLS AU, BEHR J, RICHELDI L, SCHINZEL B, et al. Nintedanib plus sildenafil in patients with idiopathic pulmonary fibrosis. N Engl J Med 2018; 379: 1722-31.

9.- OKU H, SHIMIZU T, KAWABATA T, NAGIRA M, HIKITA I, UEYAMA A, et al. Antifibrotic action of pirfenidone and prednisolone: different effects on pulmonary cytokines and growth factors in bleomycininduced murine pulmonary fibrosis. Eur J Pharmacol 2008; 590: 400-8.

10.- WOLLIN L, MAILLET I, QUESNIAUX V, HOLWEG A, RYFELL B. Antifibrotic and anti-inflammatory activity of the tyrosine kinase inhibitor nintedanib in experimental models of lung fibrosis. J Pharmacol Exp Med 2014; 349: 209-20.

11.- RUSSELL AM, ADAMALI H, MOLYNEAUX PL, LUKEY PT, MARSHALL RP, RENZONI EA, et al. Daily home spirometry: an effective tool for detecting progression in idiopathic pulmonary fibrosis. Am J Respir Crit Care Med 2016; 194: 989-97.

12.- RAGHU G. Idiopathic pulmonary fibrosis: lessons from clinical trials over the past 25 years. Eur Respir J 2017; 50: 1701209.

13.- RICHELDI L, DU BOIS RM, RAGHU G, AZUMA A, BROWN KK, COSTABEL U, et al. Efficacy and safety of nintedanib in idiopathic pulmonary fibrosis. $\mathrm{N}$ Engl J Med 2014; 370: 2071-82.

14.- KING TE JR, BRADFORD WZ, CASTROBERNARDINI S, FAGAN EA, GLASPOLE I, GLASSBERG MK, et al. A phase 3 trial of pirfenidone in patients with idiopathic pulmonary fibrosis. N Engl J Med 2014; 370: 2083-92.

15.- COSTABEL U, INOUE Y, RICHELDI L, COLLARD HR, TSCHOEPE I, STOWASSER S, et al. Efficacy of nintedanib in idiopathic pulmonary fibrosis across prespecified subgroups in INPULSIS. Am J Respir Crit Care Med 2016; 193: 178-85.

16.- NOBLE PW, ALBERA C, BRADFORD WZ, COSTABEL U, DU BOIS RM, FAGAN EA, et al. Pirfenidone for idiopathic pulmonary fibrosis: analysis of pooled data from three multinational phase 3 trials. Eur Respir J 2016; 47: 243-53.

17.- AlBERA C, COSTABEL U, FAGAN EA, GLASSBERG MK, GORINA E, LANCASTER L, et al. Efficacy of pirfenidone in patients with idiopathic pulmonary fibrosis with more preserved lung function. Eur Respir J 2016; 48: 843-51.

18.- KOLB M, RICHELDI L, BEHR J, MAHER TM, 
TANG W, STOWASSER S, et al. Nintedanib in patients with pulmonary fibrosis and preserved lung volume. Thorax 2017; 72: 340-46.

19.- WUYTS WA, KOLB M, STOWASSER S, STANSEN W, HUGGINS JT, RAGHU R. First data on efficacy and safety of nintedanib in patients with idiopathic pulmonary fibrosis and forced vital capacity of $\leq 50 \%$ of predicted value. Lung 2016; 194: 739-43.

20.- COSTABEL U, ALBERA C, GLASSBERG MK, LANCASTER LH, WUYTS WA, PETZINGER U, et al. Effect of pirfenidone in patients with more advanced idiopathic pulmonary fibrosis. Respir Res 2019; 20: 55.

21.- GLASPOLE IN, CHAPMAN SA, COOPER WA, ELLIS SJ, GOH NS, HOPKINS PM, et al. Healthrelated quality of life in idiopathic pulmonary fibrosis: data from the Australian IPFR. Respirology 2017; 22: 950-6.

22.- MAHER TM, SWIGRIS JJ, KREUTER M, WIJSENBEEK M, CASSIDY N, IRELAND L, et al. Identifying barriers to idiopathic pulmonary fibrosis treatment: a survey of patient and physician views. Respiration 2019; 96: 514-24.

23.- CARO F, BUENDÍA ROLDÁN I, CURBELO P, KAIRALLA R, MEJÍA M, NORIEGA L, et al. Grupo de estudio REFIPI. Tratamiento de la Fibrosis Pulmonar Idiopática en la vida real: Resultados parciales del Registro Latinoamericano. In: Poster presented at the Asociacion Latinoamericana de Torax Congress; 2019.

24.- CORTE T, BONELLA F, CRESTANI B, DEMEDTS MG, RICHELDI L, COECK C, et al. Safety, tolerability and appropriate use of nintedanib in idiopathic pulmonary fibrosis. Respir Res 2015; 16: 116.

25.- LANCASTER LH, DE ANDRADE JA, ZIBRAK JD, PADILLA ML, ALBERA C, NATHAN SD, et al. Pirfenidone safety and adverse event management in idiopathic pulmonary fibrosis. Eur Respir Rev 2017; 26 (146): pii: 170057.

26.- VANCHERI C, KREUTER M, RICHELDI L, RYERSON CJ, VALEYRE D, GRUTTERS JC, et al. Nintedanib with add-on pirfenidone in idiopathic pulmonary fibrosis. Results of the INJOURNEY trial. Am J Respir Crit Care Med 2018; 197: 356-63.

27.- LOVEMAN E, COPLEY VR, SCOTT DA, COLQUITT JL, CLEGG AJ, O’REILLY KM. Comparing new treatments for idiopathic pulmonary fibrosis - a network meta-analysis. BMC Pulmonary Medicine 2015; 15 (1): 37.

28.- LOVEMAN E, COPLEY VR, COLQUITT JL, SCOTT DA, CLEGG AJ, JONES J, et al. The effectiveness and cost-effectiveness of treatments for idiopathic pulmonary fibrosis: systematic review, network metaanalysis and health economic evaluation. BMC Infect Dis 2014; 15: 63. Epub 2014/11/21.

29.- LOVEMAN E, COPLEY VR, COLQUITT J, SCOTT DA, CLEGG A, JONES J, et al. The clinical effectiveness and cost-effectiveness of treatments for idiopathic pulmonary fibrosis: a systematic review and economic evaluation. Health Technol Assess 2015; 19 (20): i-xxiv, 1-336. Epub 2015/03/12.

30.- COOPER K, MENDES D, PICOT J, LOVEMAN E. Pirfenidone for the treatment of idiopathic pulmonary fibrosis: A Single Technology Appraisal. SHTAC. 2012.

31.- BAJWAH S, ROSS JR, PEACOCK JL, HIGGINSON IJ, WELLS AU, PATEL AS, et al. Interventions to improve symptoms and quality of life of patients with fibrotic interstitial lung disease: a systematic review of the literature. Thorax 2013; 68 (9): 867-79.

32.- SPAGNOLO P, DEL GIOVANE C, LUPPI F, CERRI S, BALDUZZI S, WALTERS EH, et al. Non-steroid agents for idiopathic pulmonary fibrosis. Cochrane database of systematic reviews (Online) 2010 (9): CD003134.

33.- ROCHWERG B, NEUPANE B, ZHANG Y, GARCÍA CC, RAGHU G, RICHELDI L, et al. Treatment of idiopathic pulmonary fibrosis: a network meta-analysis. BMC medicine 2016; 14 (1): 18.

34.- ARAVENA C, LABARCA G, VENEGAS C, ARENAS A, RADA G. Pirfenidone for Idiopathic Pulmonary Fibrosis: A Systematic Review and Meta-Analysis. PLoS One 2015; 10 (8): e0136160.

35.- POTTS J, YOGARATNAM D. Pirfenidone: a novel agent for the treatment of idiopathic pulmonary fibrosis. Ann Pharmacother 2013; 47 (3): 361-7.

36.- ATKINS CP, LOKE YK, WILSON AM. Outcomes in idiopathic pulmonary fibrosis: a meta-analysis from placebo controlled trials. Respir Med 2014; 108 (2): 376-87.

37.- CANESTARO WJ, FORRESTER SH, RAGHU G, HO L, DEVINE BE. Drug Treatment of Idiopathic Pulmonary Fibrosis: Systematic Review and Network Meta-Analysis. Chest 2016; 149 (3): 756-66. Epub 2016/02/03.

38.- IQWIG. [Pirfenidone - Benefit assessment according to §35a Social Code Book V (dossier assessment)]. 2011 2011. Report No.

39.- ROGLIANI P, CALZETTA L, CAVALLI F, MATERA MG, CAZZOLA M. Pirfenidone, nintedanib and $\mathrm{N}$-acetylcysteine for the treatment of idiopathic pulmonary fibrosis: A systematic review and meta-analysis. Pulm Pharmacol Ther 2016; 40: 95-103.

40.- JIANG C, HUANG H, LIU J, WANG Y, LU Z, XU $Z$. Adverse events of pirfenidone for the treatment of pulmonary fibrosis: a meta-analysis of randomized controlled trials. PLoS One 2012; 7 (10): e47024.

41.- FLEETWOOD K, MCCOOL R, GLANVILLE J, EDWARDS SC, GSTEIGER S, DAIGL M, et al. Systematic Review and Network Meta-analysis of Idiopathic Pulmonary Fibrosis Treatments. J Manag Care Spec Pharm 2017; 23 (3-b Suppl): S5-S16. Epub 2017/03/14

42.- NOBLE PW, ALBERA C, BRADFORD WZ, COSTABEL U, GLASSBERG MK, KARDATZKE D. 
Pirfenidone in patients with idiopathic pulmonary fibrosis (CAPACITY): two randomised trials. Lancet. $2011 ; 377$.

43.- AZUMA A, NUKIWA T, TSUBOI E, SUGA M, ABE S, NAKATA K. Double-blind, placebo-controlled trial of pirfenidone in patients with idiopathic pulmonary fibrosis. Am J Respir Crit Care Med. 2005; 171: 1040-7.
44.- TANIGUCHI H, EBINA M, KONDOH Y, OGURA T, AZUMA A, SUGA M. Pirfenidone in idiopathic pulmonary fibrosis. Eur Respir J 2010; 35: 821-9.

45.- RICHELDI L, COSTABEL U, SELMAN M, KIM DS, HANSELL DM, NICHOLSON AG, et al. Efficacy of a tyrosine kinase inhibitor in idiopathic pulmonary fibrosis. N Engl J Med 2011; 365: 1079-87. 